\title{
Twistorial Foundation for the Classical Double Copy
}

\author{
Chris D. White \\ Centre for Research in String Theory, School of Physics and Astronomy, Queen Mary University of London, \\ 327 Mile End Road, London E1 4NS, United Kingdom
}

(Received 4 October 2020; revised 4 December 2020; accepted 13 January 2021; published 12 February 2021)

\begin{abstract}
The classical double copy relates exact solutions of gauge, gravity, and other theories. Although widely studied, its origins and domain of applicability have remained mysterious. In this Letter, I show that a particular incarnation - the Weyl double copy—can be derived using well-established ideas from twistor theory. As well as explaining where the Weyl double copy comes from, the twistor formalism also shows that it is more general than previously thought.
\end{abstract}

DOI: 10.1103/PhysRevLett.126.061602

Introduction.-The study of fundamental physics is dominated by (non-Abelian) gauge theories, which underly particle physics, and general relativity (GR), which describes astrophysics and cosmology. Intriguing similarities between these theories have emerged in recent years, and I will here concentrate on the classical double copy that provides a map between solutions of different field equations, itself inspired by a similar procedure for (quantum) scattering amplitudes $[1,2]$. In general, one can only relate classical solutions at a fixed order in perturbation theory [3-38]. However, in some cases it is possible to make statements about exact solutions [39-69]. In particular, the Kerr-Schild double copy of Ref. [39] relates certain algebraically special spacetimes in GR to an exact solution of the gauge theory field equations. The latter is referred to as the single copy of the given gravity solution, and there is also a zeroth copy, which produces a solution of so-called biadjoint scalar field theory. A second exact classical double copy is the recent Weyl double copy of Ref. [50], which I discuss below. This relies on a well-known formalism (see, e.g., Refs. [70-72]) which recasts the usual tensorial field equations for electromagnetism and GR in terms of two-component spinors $\pi_{A}$ and their complex conjugates $\pi_{A^{\prime}}$. Every spacetime index of a tensor field can be converted to a pair of spinorial indices (and vice versa) by contracting with Infeld-van der Waerden symbols $\sigma_{A A^{\prime}}^{\mu}$. Furthermore, spinors with multiple (un)primed indices can always be decomposed into sums of products of LeviCivita symbols and fully symmetric spinors. For example, the field strength tensor of electromagnetism has the spinorial translation

Published by the American Physical Society under the terms of the Creative Commons Attribution 4.0 International license. Further distribution of this work must maintain attribution to the author(s) and the published article's title, journal citation, and DOI. Funded by SCOAP ${ }^{3}$.

$$
F_{\alpha \beta} \rightarrow F_{A A^{\prime} B B^{\prime}}=\phi_{A B} \epsilon_{A^{\prime} B^{\prime}}+\bar{\phi}_{A B} \epsilon_{A^{\prime} B^{\prime}},
$$

where the symmetric spinors $\phi_{A B}$ and $\bar{\phi}_{A^{\prime} B^{\prime}}$ turn out to correspond separately to the anti-self-dual and self-dual parts in spacetime. In GR, we will be concerned with vacuum spacetimes, for which the Riemann tensor reduces to the Weyl tensor, with spinorial translation

$$
C_{\alpha \beta \gamma \delta} \rightarrow \Psi_{A B C D} \epsilon_{A^{\prime} B^{\prime}} \epsilon_{C^{\prime} D^{\prime}}+\bar{\Psi}_{A^{\prime} B^{\prime} C^{\prime} D^{\prime}} \epsilon_{A B} \epsilon_{C D}
$$

such that $\Psi_{A B C D}\left(\bar{\Psi}_{A^{\prime} B^{\prime} C^{\prime} D^{\prime}}\right)$ is the self-dual (anti-self-dual) part, and the former is called the Weyl spinor. The vacuum electromagnetic and GR equations are special cases of the general massless free-field equation

$$
\nabla^{A A^{\prime}} \phi_{A B \ldots E}=0, \quad \nabla^{A A^{\prime}} \bar{\phi}_{A^{\prime} B^{\prime} \ldots E^{\prime}}=0
$$

where $\phi_{A B \ldots E}$ is assumed symmetric, with $2 s$ indices for a field of spin $s$, and $\nabla^{A A^{\prime}}$ is the appropriate spinorial translation of the spacetime covariant derivative. Any symmetric spinor factorises into a symmetrized product of one-index principal spinors, allowing one to classify solutions of different theories. Electromagnetic spinors are null (non-null) if their principal spinors are (non)proportional. Weyl spinors with no common principal spinors are called Petrov type I, and the possible patterns of degeneracy $\{2,1,1\},\{3,1\},\{2,2\}$, and $\{4\}$ are types II, III, D, and N, respectively.

Given two (possibly equal) electromagnetic field strength spinors $\phi_{A B}$ and $\tilde{\phi}_{A B}$, the Weyl double copy of Ref. [50] states that one may construct a Weyl spinor according to the rule

$$
\Psi_{A B C D}=\frac{1}{S} \phi_{(A B} \tilde{\phi}_{C D)}
$$

where $S(x)$ is a scalar field. This procedure was argued to hold for arbitrary type D vacuum spacetimes in Ref. [50], 
where the scalar $S$ could then be found in particular examples by matching both sides of Eq. (4). All of these solutions have the property that they linearize the Einstein equations, so that the derivative in Eq. (3) may be taken to be in flat space.

Applications of the double copy range across many different areas of physics, including new methods for investigating gravitational waves and/or insights into quantum gravity (see, e.g., Ref. [73]), connections between gauge and gravity theories and fluid dynamics [61,74], relations between novel optical systems and gravity $[75,76]$, and studies of magnetic monopoles and topology [54]. But quite how general the double copy is, and where it ultimately comes from, have up until now remained mysterious [77], with many open questions: (i) Why is it possible to formulate an exact double copy in position space, when the original procedure for amplitudes [1,2] is in momentum space? (ii) How can one systematically fix the scalar function $S(x)$, and is there a well-defined procedure for the inverse zeroth copy that relates this to a gauge theory solution? (iii) Can one generalize the Weyl or Kerr-Schild double copies to curved spacetime (see Refs. [42,43,79] for related work)? (iv) Can one generalize the Weyl double copy to less algebraically special cases (i.e., other Petrov types)? We will be able to answer all of these questions, by traveling to twistor space. Twistor methods have been highly successful in the study of scattering amplitudes (see, e.g., Refs. [80-84]). The present study, however, constitutes the first application to the exact double copy of classical solutions.

Twistor space and Penrose transforms.-Twistor space (see, e.g., Refs. [71,85,86]) T corresponds to the set of solutions of the twistor equation

$$
\nabla_{A^{\prime}}^{(A} \Omega^{B)}=0 \Rightarrow \Omega^{A}=\omega^{A}-i x^{A A^{\prime}} \pi_{A^{\prime}},
$$

where the second equation gives the general solution in Minkowski space. We may thus associate solutions of Eq. (5) with four-component objects ("twistors") containing a pair of spinors:

$$
Z^{\alpha}=\left(\omega^{A}, \pi_{A^{\prime}}\right)
$$

whose Minkowski space "location" is defined by $\Omega^{A}=0$. From Eq. (5), this implies the incidence relation

$$
\omega^{A}=i x^{A A^{\prime}} \pi_{A^{\prime}},
$$

which is invariant under rescalings $Z^{\alpha} \rightarrow \lambda Z^{\alpha}$. Thus, twistors obeying Eq. (7) are points in projective twistor space $\mathbb{P T}$. A fixed point $x^{\mu}$ in Minkowski space maps to a complex line in $\mathbb{P} \mathbb{T}$, corresponding to the celestial sphere of null directions at $x^{\mu}$. Considering the conjugate equation to Eq. (5), we may also define dual twistors $W_{\alpha}$ and an inner product $Z^{\alpha} W_{\alpha}$, which turns out to be conformally invariant. Then the Penrose transform

$\phi_{A^{\prime} B^{\prime} \ldots C^{\prime}}(x)=\frac{1}{2 \pi i} \oint_{\Gamma} \pi_{E^{\prime}} d \pi^{E^{\prime}} \pi_{A^{\prime}} \pi_{B^{\prime}} \ldots \pi_{C^{\prime}}\left[\rho_{x} f\left(Z^{\alpha}\right)\right]$

relates holomorphic twistor functions $f\left(Z^{\alpha}\right)$ in $\mathbb{P} \mathbb{T}$ (i.e., involving no nonconstant dual twistors) with spacetime fields, where $\rho_{x}$ denotes restriction to the celestial sphere of spacetime point $x^{A A^{\prime}}$ in $\mathbb{P \mathbb { T }}$ and $\Gamma$ is an arbitrary contour separating any poles. For consistency, the function $f\left(Z^{\alpha}\right)$ must be homogeneous under twistor rescalings with degree $(-n-2)$, where $n$ is the number of indices appearing on the left-hand side. Equation (8) solves the spin- $n$ massless field equation of Eq. (3), except for the scalar case, which instead satisfies the conformally invariant wave equation with Ricci scalar $R$ :

$$
\left(\square+\frac{R}{6}\right) \phi=0
$$

The Weyl double copy from twistor space.-I can now state the main result of my Letter, namely, a derivation of the Weyl double copy from twistor space. Consider a pair of homogeneity-4 twistor functions $\left\{f_{\mathrm{EM}}^{(1,2)}\right\}$, which will necessarily correspond to electromagnetic solutions $\phi_{A^{\prime} B^{\prime}}^{(1,2)}$ in spacetime by Eq. (8). One may then combine them with a homogeneity- 2 function $f_{\text {scal }}$ (corresponding to a spacetime scalar $\phi$ ) to make a homogeneity-6 function according to

$$
f_{\text {grav }}=\frac{f_{\mathrm{EM}}^{(1)} f_{\mathrm{EM}}^{(2)}}{f_{\mathrm{scal}}} .
$$

This leads to a (linearized) gravity solution $\phi_{A^{\prime} B^{\prime} C^{\prime} D^{\prime}}$ in spacetime, implying that there will be some sort of spacetime relationship between electromagnetic, scalar, and gravitational fields. My claim is that, for suitable functions, this is precisely the mixed Weyl double copy [87]

$$
\phi_{A^{\prime} B^{\prime} C^{\prime} D^{\prime}}=\frac{\phi_{\left(A^{\prime} B^{\prime}\right.}^{(1)} \phi_{\left.C^{\prime} D^{\prime}\right)}^{(2)}}{\phi} .
$$

I have here synchronized my notation with Eq. (3), but $\phi_{A^{\prime} B^{\prime} C^{\prime} D^{\prime}}$ and $\phi$ are the conjugates of the quantities $\Psi_{A B C D}$ and $S$, respectively, appearing in Eq. (4). In order to determine the functions we must use in Eq. (10), we may rely on the observation that if a twistor function has an $m$ th-order pole, its corresponding spacetime field has an $(n-m+1)$-fold principal spinor, where $n$ is the number of spinor indices (see, e.g., Ref. [71]). We may then choose the function $f_{\text {scal }}$ to have poles at the same locations in twistor space as the functions $f_{\mathrm{EM}}$ and $f_{\text {grav }}$, and we may choose the order of the poles in each case so as to reproduce Eq. (11) in spacetime. To see how this works, consider the functions 
$f_{m}=\frac{\left[Q_{\alpha \beta} Z^{\alpha} Z^{\beta}\right]^{-m}}{m !} \equiv \frac{1}{m !}\left[\frac{N^{-1}(x)}{\left[\xi-\xi_{1}(x)\right]\left[\xi-\xi_{2}(x)\right]}\right]^{-m}$,

for some constant dual twistor $Q_{\alpha \beta}$, where $m=1,2$, and 3 for the scalar, electromagnetic, and gravity cases, respectively. In the second equation, I have chosen homogeneous coordinates $\pi_{A^{\prime}}=(1, \xi), \xi \in \mathbb{C}$. The resulting roots in $\xi$ and normalization factor $N(x)$ gain their position dependence from the use of the incidence relation of Eq. (7). One then finds that the Penrose transform in the general spin $(m-1)$ case is

$$
\underbrace{\phi_{A^{\prime} B^{\prime} \ldots C^{\prime}}}_{(m-1) \text { indices }}=\frac{N^{m}(x)}{2 \pi i} \oint_{\Gamma} d \xi \overbrace{\frac{(1, \xi)_{A^{\prime}}(1, \xi)_{B^{\prime}} \ldots(1, \xi)_{C^{\prime}}}{(\xi-1) \text { factors }}}^{\left(\xi-\xi_{1}\right)^{m}\left(\xi-\xi_{2}\right)^{m}} .
$$

The contour $\Gamma$ is defined on the Riemann sphere of $\xi$ and is such that it must separate the poles at $\xi=\xi_{1}$ and $\xi=\xi_{2}$. Choosing to enclose the first of these poles, one finds spacetime fields

$$
\begin{aligned}
\phi & =\frac{N(x)}{\xi_{1}-\xi_{2}}, \quad \phi_{A^{\prime} B^{\prime}}=-\frac{N^{2}(x)}{\left(\xi_{1}-\xi_{2}\right)^{3}} \alpha_{\left(A^{\prime}\right.} \beta_{\left.B^{\prime}\right)}, \\
\phi_{A^{\prime} B^{\prime} C^{\prime} D^{\prime}} & =\frac{N^{3}(x)}{\left(\xi_{1}-\xi_{2}\right)^{5}} \alpha_{\left(A^{\prime}\right.} \beta_{B^{\prime}} \alpha_{C^{\prime}} \beta_{\left.D^{\prime}\right)},
\end{aligned}
$$

where the principal spinors occurring in these equations are given by

$$
\alpha=\left(1, \xi_{1}\right), \quad \beta=\left(1, \xi_{2}\right) .
$$

It is evident that the fields of Eq. (14) obey Eq. (11), as required. Furthermore, the gravity field in Eq. (14) is clearly of type $\mathrm{D}$, where the right pattern $(2,2)$ of degenerate principal spinors arises from choosing two distinct third-order poles for $f_{\text {grav }}$ in twistor space.

To illustrate the above, we may consider a simple special case of Eq. (12), namely, the self-dual SchwarzschildTaub-NUT solution, which is generated by choosing [88]

$$
Q_{\alpha \beta}=\frac{1}{2}\left(\begin{array}{rrrr}
0 & 0 & 0 & -1 \\
0 & 0 & 1 & 0 \\
0 & 1 & 0 & 0 \\
-1 & 0 & 0 & 0
\end{array}\right) .
$$

One then finds

$$
\xi_{1,2}=\frac{-z \pm r}{(x+i y)}=\frac{x-i y}{z \pm r}, \quad N(x)=\frac{i \sqrt{2}}{(x+i y)},
$$

where $r=\sqrt{x^{2}+y^{2}+z^{2}}$ is the spherical radial coordinate. From Eq. (14), the biadjoint scalar function $\phi$ associated with this solution is given by

$$
\phi=\frac{i}{r \sqrt{2}} .
$$

This agrees with the function $S(x)$ presented in Ref. [50], up to an overall normalization constant. However, their function $S(x)$ is itself defined only up to an overall constant, so this is not a problem. Furthermore, given the principal spinors of Eqs. (15) and (17), one may convert to the Kerr-Schild form of the classical double copy by contracting each with the relevant Infeld-van der Waerden symbols [70] to obtain the spacetime vectors:

$$
k_{\mu}^{ \pm} \propto\left(1, \pm \frac{x}{r}, \pm \frac{y}{r}, \pm \frac{z}{r}\right) .
$$

These are indeed the two possible choices of null vector entering the Kerr-Schild double copy approach of Ref. [39].

In general, the Weyl double copy becomes especially elegant in twistor space: It relies on simple products of scalar functions, whereas the spacetime fields involve products of lower-rank spinors followed by symmetrization over indices. However, the twistor space formulation is much more than a simple rewriting. Once one has found the set of functions in Eq. (12) for use in Eq. (10), the known properties of the Penrose transform guarantee that there exist corresponding spacetime fields and that these obey the Weyl double copy. Furthermore, the functional form of Eq. (12) is sufficient to produce the self-dual part of the most general type D vacuum solution $[89,90]$ and, thus, encompasses the solutions considered in Ref. [50]. Hence, the twistor framework provides a derivation of both the form and the previously considered scope of the Weyl double copy. In doing so, it also explains why the Weyl double copy (and its related Kerr-Schild counterpart) operate directly in position space, as it is the latter that arises from the Penrose transform.

My argument here is confined to linearized equations of motion only, due to the limitations of the Penrose transform. However, for the type $\mathrm{D}$ vacuum solutions considered in Ref. [50], all of them linearize their respective field equations, so that the Weyl double copy can be promoted to an exact statement. It may also be generalized, in principle, to arbitrary conformally flat background spacetimes, formalizing previous exploratory work in this direction $[42,43]$. To see this, note that the twistor description is manifestly conformally invariant (as stated above), so that, upon obtaining the spacetime fields of Eq. (14), one may transform each one to a given background spacetime according to the usual conformal transformation rules for multi-index spinors [71]. The resulting fields can then be interpreted as related by the double copy in the new background.

The inverse zeroth copy.-As mentioned above, it has not previously been clear how to fix the scalar function that 
appears in the Weyl double copy. In both this and the KerrSchild approach, it is also not obvious how to precisely formulate an inverse zeroth copy that relates a given scalar field to corresponding gauge and gravity solutions. The latter contain extra kinematic information (associated with the principal null directions of the field strength and Weyl tensor, respectively) that appears entirely absent in the biadjoint theory. The twistor approach solves this problem: The principal null directions of the gauge and gravity fields are uniquely fixed by the poles of the corresponding twistor space functions, as evidenced directly in Eq. (15). What is more, this information is already present in the scalar function $(m=1)$ of Eq. (12). The twistor picture thus reveals, for the first time, how the biadjoint field "knows" about the structure of the resulting gauge and gravity fields.

Beyond type D solutions.- So far, we have reproduced the type D Weyl double copy of Ref. [50], by choosing a particular set of functions [Eq. (12)] for use in Eq. (10). However, we can clearly allow for a more general set of functions to be used, and in doing so the twistor language allows us to extend the Weyl double copy to solutions other than Petrov type D.

To find a concrete example, we may use a particularly well-studied class of holomorphic twistor functions, namely, elementary states (see, e.g., Ref. [71]), which consist of ratios of factors of the form $\left(A_{\alpha} Z^{\alpha}\right)$, where $A_{\alpha}$ is a constant dual twistor. Such functions were originally motivated as alternatives to plane-wave states in examining scattering processes via twistor space but have been reconsidered in a recent series of papers [76,91-95], where they are shown to give rise to topologically nontrivial configurations of electromagnetic and gravitational fields, where the field lines form torus knots. Knotted magnetic fields are of great interest due to their potential role in stabilizing nuclear fusion processes and for stellar structure [96]. Furthermore, finding gravitational counterparts of interesting electromagnetic solutions may guide experimental efforts to emulate gravitational waves [97].

In particular, consider the Penrose transform pair [94]

$$
\begin{gathered}
\frac{1}{\left(A_{\alpha} Z^{\alpha}\right)^{1+a}\left(B_{\alpha} Z^{\alpha}\right)^{1+b}} \rightarrow \\
\left(\frac{2}{\Omega|x-y|^{2}}\right)^{a+b+1} \mathcal{A}_{\left(A_{1}^{\prime}\right.} \ldots \mathcal{A}_{A_{b}^{\prime}} \mathcal{B}_{A_{b+1}^{\prime}} \ldots \mathcal{B}_{A_{2 h}^{\prime}},
\end{gathered}
$$

where $a, b \in \mathbb{Z}$, the curly spinors are defined by

$$
A_{\alpha} Z^{\alpha} \equiv \mathcal{A}^{A^{\prime}} \pi_{A^{\prime}}, \quad B_{\beta} Z^{\beta} \equiv \mathcal{B}^{B^{\prime}} \pi_{B^{\prime}},
$$

and

$$
\begin{aligned}
& A_{\alpha}=\left(\mu_{A}, \lambda^{A^{\prime}}\right), \quad B_{\alpha}=\left(\sigma_{A}, \psi^{A^{\prime}}\right), \\
& \Omega=\mu_{B} \sigma^{B}, \quad y^{A A^{\prime}}=i \frac{\sigma^{A} \lambda^{A^{\prime}}-\mu^{A} \psi^{A^{\prime}}}{\mu_{B} \sigma^{B}} .
\end{aligned}
$$

For $a=b=0$, we obtain the scalar field

$$
\phi=\frac{2}{\Omega|x-y|^{2}} .
$$

One may construct twistor functions of homogeneity 4 by choosing $(a, b)=(1,1)$ or $(0,2)$, leading to the two respective electromagnetic spinors

$$
\begin{aligned}
& \phi_{A^{\prime} B^{\prime}}^{(1,1)}=\left(\frac{2}{\Omega|x-y|^{2}}\right)^{3} \mathcal{A}_{A^{\prime}} \mathcal{B}_{B^{\prime}}, \\
& \phi_{A^{\prime} B^{\prime}}^{(0,2)}=\left(\frac{2}{\Omega|x-y|^{2}}\right)^{3} \mathcal{A}_{A^{\prime}} \mathcal{A}_{B^{\prime}} .
\end{aligned}
$$

Using these in the mixed Weyl double copy of Eq. (11), one can generate a number of different Weyl spinors:

$$
\begin{gathered}
\phi_{A^{\prime} B^{\prime} C^{\prime} D^{\prime}}^{(1,1) \times(1,1)}=\left(\frac{2}{\Omega|x-y|^{2}}\right)^{5} \mathcal{A}_{\left(A^{\prime}\right.} \mathcal{A}_{B^{\prime}} \mathcal{B}_{C^{\prime}} \mathcal{B}_{\left.D^{\prime}\right)}, \\
\phi_{A^{\prime} B^{\prime} C^{\prime} D^{\prime}}^{(1,1) \times(0,2)}=\left(\frac{2}{\Omega|x-y|^{2}}\right)^{5} \mathcal{A}_{\left(A^{\prime}\right.} \mathcal{A}_{B^{\prime}} \mathcal{A}_{C^{\prime}} \mathcal{B}_{\left.D^{\prime}\right)}, \\
\phi_{A^{\prime} B^{\prime} C^{\prime} D^{\prime}}^{(0,2) \times(0,2)}=\left(\frac{2}{\Omega|x-y|^{2}}\right)^{5} \mathcal{A}_{\left(A^{\prime}\right.} \mathcal{A}_{B^{\prime}} \mathcal{A}_{C^{\prime}} \mathcal{A}_{\left.D^{\prime}\right)},
\end{gathered}
$$

and it is easily checked that these are the fields that arise upon multiplying the corresponding functions in twistor space according to Eq. (10) and then performing the Penrose transform to position space. The first and third of these examples are Petrov type D and N, respectively. However, the second (as already noted in Ref. [94]) is Petrov type III. This thus goes beyond the original formulation of the Weyl double copy in Ref. [50]. The price one pays, however, is that such solutions are restricted to linear level only.

Conclusion.-I have presented a twistor space derivation of the exact classical double copy that reproduces the Weyl double copy of Ref. [50], itself equivalent to the KerrSchild double copy of Ref. [39], where they overlap. It resolves a number of questions, which I labeled above: (i) the Penrose transform of Eq. (8) relates twistor functions to spacetime fields in position space, thus explaining why a position space exact copy is possible; (ii) the scalar function $S(x)$ in Eq. (4) is predicted exactly by the twistor approach, and its poles in twistor space already know what the principal spinors in the gauge and gravity solutions will be, thus providing an explicit interpretation for the inverse zeroth copy; (iii) conformal invariance of the twistor space formulation implies that the classical double copy should immediately generalize to conformally flat spacetimes, 
formalizing the exploratory results of Refs. $[42,43]$, and where the biadjoint field should obey Eq. (9); (iv) my new approach leads to Petrov types other than type D (or N), thereby broadening the scope of the Weyl double copy.

I expect that the twistor language could have a number of uses, including providing new explicit examples of the classical double copy and to ascertain its scope [e.g., by showing which Petrov types are (not) possible]. In line with the general remarks above, it would be interesting to formulate explicit examples of double copies in conformally flat backgrounds, including those of astrophysical relevance. I also note that the twistor language can, in principle, be extended beyond linear level, using appropriate generalizations of the Penrose transform $[98,99]$.

My methods have been manifestly four dimensional. For higher dimensions, it may be more sensible to use an ambitwistor approach in which (dual) twistors are placed on a more equal footing, as has proven useful for scattering amplitudes [80-84]. In any case, given the role that twistor theory has played in many different areas of physics and mathematics [100], I hope that this Letter attracts the interest of communities who have been previously unaware of the fascinating subject of the double copy.

This work has been supported by the United Kingdom Science and Technology Facilities Council (STFC) Consolidated Grant No. ST/P000754/1 "String theory, gauge theory and duality" and by the European Union Horizon 2020 research and innovation program under the Marie Skłodowska-Curie Grant Agreement No. 764850 "SAGEX."

[1] Z. Bern, J. J. M. Carrasco, and H. Johansson, Phys. Rev. Lett. 105, 061602 (2010).

[2] Z. Bern, T. Dennen, Y.-t. Huang, and M. Kiermaier, Phys. Rev. D 82, 065003 (2010).

[3] A. Luna, R. Monteiro, I. Nicholson, A. Ochirov, D. O'Connell, N. Westerberg, and C. D. White, J. High Energy Phys. 04 (2017) 069.

[4] W. D. Goldberger and A. K. Ridgway, Phys. Rev. D 95, 125010 (2017).

[5] W. D. Goldberger, S. G. Prabhu, and J. O. Thompson, Phys. Rev. D 96, 065009 (2017).

[6] W. D. Goldberger and A. K. Ridgway, Phys. Rev. D 97, 085019 (2018).

[7] W. D. Goldberger, J. Li, and S. G. Prabhu, Phys. Rev. D 97, 105018 (2018).

[8] C.-H. Shen, J. High Energy Phys. 11 (2018) 162.

[9] M. Carrillo-Gonzalez, R. Penco, and M. Trodden, J. High Energy Phys. 11 (2018) 065.

[10] J. Plefka, J. Steinhoff, and W. Wormsbecher, Phys. Rev. D 99, 024021 (2019).

[11] J. Plefka, C. Shi, J. Steinhoff, and T. Wang, Phys. Rev. D 100, 086006 (2019).

[12] W. D. Goldberger and J. Li, J. High Energy Phys. 02 (2020) 092.
[13] A. PV and A. Manu, Phys. Rev. D 101, 046014 (2020).

[14] A. Anastasiou, L. Borsten, M. J. Duff, L. J. Hughes, and S. Nagy, Phys. Rev. Lett. 113, 231606 (2014).

[15] L. Borsten and M. J. Duff, Phys. Scr. 90, 108012 (2015).

[16] A. Anastasiou, L. Borsten, M. J. Duff, M. J. Hughes, A. Marrani, S. Nagy, and M. Zoccali, Phys. Rev. D 96, 026013 (2017).

[17] G. L. Cardoso, S. Nagy, and S. Nampuri, J. High Energy Phys. 10 (2016) 127.

[18] L. Borsten, Phys. Rev. D 97, 066014 (2018).

[19] A. Anastasiou, L. Borsten, M. J. Duff, A. Marrani, S. Nagy, and M. Zoccali, Contemp. Math. 721, 1 (2019).

[20] A. Anastasiou, L. Borsten, M. J. Duff, S. Nagy, and M. Zoccali, Phys. Rev. Lett. 121, 211601 (2018).

[21] G. Cardoso, G. Inverso, S. Nagy, and S. Nampuri, Proc. Sci 318, 177 (2018).

[22] A. Luna, S. Nagy, and C. White, J. High Energy Phys. 09 (2020) 062.

[23] L. Borsten and S. Nagy, J. High Energy Phys. 07 (2020) 093.

[24] L. Borsten, B. Juřco, H. Kim, T. Macrelli, C. Saemann, and M. Wolf, arXiv:2007.13803.

[25] A. Luna, I. Nicholson, D. O'Connell, and C. D. White, J. High Energy Phys. 03 (2018) 044.

[26] D. A. Kosower, B. Maybee, and D. O'Connell, J. High Energy Phys. 02 (2019) 137.

[27] B. Maybee, D. O'Connell, and J. Vines, J. High Energy Phys. 12 (2019) 156.

[28] Y. F. Bautista and A. Guevara, arXiv:1908.11349.

[29] Y. F. Bautista and A. Guevara, arXiv:1903.12419.

[30] C. Cheung, I. Z. Rothstein, and M. P. Solon, Phys. Rev. Lett. 121, 251101 (2018).

[31] Z. Bern, C. Cheung, R. Roiban, C.-H. Shen, M. P. Solon, and M. Zeng, J. High Energy Phys. 10 (2019) 206.

[32] Z. Bern, C. Cheung, R. Roiban, C.-H. Shen, M. P. Solon, and M. Zeng, Phys. Rev. Lett. 122, 201603 (2019).

[33] Z. Bern, A. Luna, R. Roiban, C.-H. Shen, and M. Zeng, arXiv:2005.03071.

[34] G. Klin and R. A. Porto, J. High Energy Phys. 01 (2020) 072.

[35] G. Klin and R. A. Porto, J. High Energy Phys. 11 (2020) 106.

[36] G. L. Almeida, S. Foffa, and R. Sturani, J. High Energy Phys. 11 (2020) 165.

[37] H. Godazgar, M. Godazgar, R. Monteiro, D. Peinador Veiga, and C. Pope, arXiv:2010.02925.

[38] E. Chacón, H. García-Compeán, A. Luna, R. Monteiro, and C. D. White, arXiv:2008.09603.

[39] R. Monteiro, D. O'Connell, and C. D. White, J. High Energy Phys. 12 (2014) 056.

[40] A. Luna, R. Monteiro, D. O'Connell, and C. D. White, Phys. Lett. B 750, 272 (2015).

[41] A. Luna, R. Monteiro, I. Nicholson, D. O'Connell, and C. D. White, J. High Energy Phys. 06 (2016) 023.

[42] M. Carrillo-Gonzlez, R. Penco, and M. Trodden, J. High Energy Phys. 04 (2018) 028.

[43] N. Bahjat-Abbas, A. Luna, and C. D. White, J. High Energy Phys. 12 (2017) 004.

[44] D. S. Berman, E. Chacn, A. Luna, and C. D. White, J. High Energy Phys. 01 (2019) 107.

[45] I. Bah, R. Dempsey, and P. Weck, J. High Energy Phys. 02 (2020) 180. 
[46] M. Carrillo Gonzlez, B. Melcher, K. Ratliff, S. Watson, and C. D. White, J. High Energy Phys. 07 (2019) 167.

[47] A. Banerjee, E. Colgin, J. A. Rosabal, and H. Yavartanoo, Phys. Rev. D 102, 126017 (2020).

[48] A. Ilderton, Phys. Lett. B 782, 22 (2018).

[49] R. Monteiro, I. Nicholson, and D. O'Connell, Classical Quantum Gravity 36, 065006 (2019).

[50] A. Luna, R. Monteiro, I. Nicholson, and D. O'Connell, Classical Quantum Gravity 36, 065003 (2019).

[51] K. Lee, J. High Energy Phys. 10 (2018) 027.

[52] W. Cho and K. Lee, J. High Energy Phys. 07 (2019) 030.

[53] K. Kim, K. Lee, R. Monteiro, I. Nicholson, and D. Peinador Veiga, J. High Energy Phys. 02 (2020) 046.

[54] L. Alfonsi, C. D. White, and S. Wikeley, J. High Energy Phys. 07 (2020) 091.

[55] N. Bahjat-Abbas, R. Stark-Mucho, and C. D. White, J. High Energy Phys. 04 (2020) 102.

[56] C. D. White, Phys. Lett. B 763, 365 (2016).

[57] P.-J. De Smet and C. D. White, Phys. Lett. B 775, 163 (2017).

[58] N. Bahjat-Abbas, R. Stark-Mucho, and C. D. White, Phys. Lett. B 788, 274 (2019).

[59] G. Elor, K. Farnsworth, M. L. Graesser, and G. Herczeg, J. High Energy Phys. 12 (2020) 121.

[60] M. K. Gumus and G. Alkac, Phys. Rev. D 102, 024074 (2020).

[61] C. Keeler, T. Manton, and N. Monga, J. High Energy Phys. 08 (2020) 147.

[62] N. Arkani-Hamed, Y.-t. Huang, and D. O'Connell, J. High Energy Phys. 01 (2020) 046.

[63] Y.-T. Huang, U. Kol, and D. O'Connell, Phys. Rev. D 102, 046005 (2020).

[64] R. Alawadhi, D. S. Berman, B. Spence, and D. Peinador Veiga, J. High Energy Phys. 03 (2020) 059.

[65] N. Moynihan, J. High Energy Phys. 01 (2020) 014.

[66] R. Alawadhi, D. S. Berman, and B. Spence, J. High Energy Phys. 09 (2020) 127.

[67] D. A. Easson, C. Keeler, and T. Manton, Phys. Rev. D 102, 086015 (2020).

[68] E. Casali and A. Puhm, arXiv:2007.15027.

[69] A. Cristofoli, J. High Energy Phys. 11 (2020) 160.

[70] R. Penrose and W. Rindler, Spinors and SpaceTime, Cambridge Monographs on Mathematical Physics (Cambridge University Press, Cambridge, England, 2011), ISBN 978-0-521-33707-6, 978-0-511-86766-8, 978-0521-33707-6.

[71] R. Penrose and W. Rindler, Spinors and Space-Time: Spinor and Twistor Methods in Space-Time Geometry, Cambridge Monographs on Mathematical Physics Vol. 2 (Cambridge University Press, Cambridge, England, 1988), ISBN 978-0-521-34786-0, 978-0-511-86842-9.

[72] J. Stewart, Advanced General Relativity, Cambridge Monographs on Mathematical Physics (Cambridge
University Press, Cambridge, England, 1994), ISBN 978-0-521-44946-5, 978-0-511-87118-4.

[73] Z. Bern, J. J. Carrasco, M. Chiodaroli, H. Johansson, and R. Roiban, arXiv:1909.01358.

[74] C. Cheung and J. Mangan, arXiv:2010.15970.

[75] S. M. Barnett, New J. Phys. 16, 023027 (2014).

[76] S. Sabharwal and J. W. Dalhuisen, J. High Energy Phys. 07 (2019) 004.

[77] For tree-level scattering amplitudes, the double copy has a string-theoretic origin [78].

[78] H. Kawai, D. Lewellen, and S. Tye, Nucl. Phys. B269, 1 (1986).

[79] S. G. Prabhu, arXiv:2011.06588.

[80] L. Mason and D. Skinner, J. High Energy Phys. 07 (2014) 048.

[81] N. Arkani-Hamed, F. Cachazo, C. Cheung, and J. Kaplan, J. High Energy Phys. 03 (2010) 110.

[82] L. Mason and D. Skinner, J. High Energy Phys. 01 (2010) 064.

[83] Y. Geyer, A. E. Lipstein, and L. J. Mason, Phys. Rev. Lett. 113, 081602 (2014).

[84] E. Casali, Y. Geyer, L. Mason, R. Monteiro, and K. A. Roehrig, J. High Energy Phys. 11 (2015) 038.

[85] S. Huggett and K. Tod, An Introduction to Twistor Theory (Cambridge University Press, Cambridge, 1986), ISBN 978-0-521-45689-0.

[86] T. Adamo, Proc. Sci. Modave 2017 (2018) 003.

[87] I have here focused on the conjugate version of Eq. (4), so that I can use the Penrose transform of Eq. (8).

[88] I have been inspired by a similar function for anti-self-dual fields in Ref. [89].

[89] Advances in Twistor Theory, edited by L. Hughston et al. (1979).

[90] L. Haslehurst and R. Penrose, Twistor Newslett. 34, 1 (1992).

[91] J. Dalhuisen and D. Bouwmeester, J. Phys. A 45, 135201 (2012).

[92] J. Swearngin, A. Thompson, A. Wickes, J. W. Dalhuisen, and D. Bouwmeester, arXiv:1302.1431.

[93] A. J. J. M. de Klerk, R. I. van der Veen, J. W. Dalhuisen, and D. Bouwmeester, Phys. Rev. A 95, 053820 (2017).

[94] A. Thompson, A. Wickes, J. Swearngin, and D. Bouwmeester, J. Phys. A 48, 205202 (2015).

[95] A. Thompson, J. Swearngin, and D. Bouwmeester, J. Phys. A 47, 355205 (2014).

[96] M. Arrayás, D. Bouwmeester, and J. Trueba, Phys. Rep. 667, 1 (2017).

[97] I. Fernandez-Corbaton, M. Cirio, A. Büse, L. Lamata, E. Solano, and G. Molina-Terriza, Sci. Rep. 5, 11538 (2015).

[98] R. Ward, Phys. Lett. 61A, 81 (1977).

[99] R. Penrose, Gen. Relativ. Gravit. 7, 171 (1976).

[100] M. Atiyah, M. Dunajski, and L. Mason, Proc. R. Soc. A 473, 20170530 (2017). 